九州大学学術情報リポジトリ

Kyushu University Institutional Repository

\title{
ESTIMATING THE CORRELATION DIMENSION FROM CHAOTIC DYNAMICAL SYSTEMS BY U-STATISTICS
}

\section{Kawaguchi, Atsushi}

Graduate School of Mathematics, Kyushu University

https://doi.org/10.5109/13515

出版情報: Bulletin of informatics and cybernetics. 34 (2), pp.143-150, 2002-12. Research Association of Statistical Sciences バージョン:

権利関係 : 


\title{
ESTIMATING THE CORRELATION DIMENSION FROM CHAOTIC DYNAMICAL SYSTEMS BY U-STATISTICS
}

\author{
By
}

\section{Atsushi Kawagugh*}

\begin{abstract}
An estimator of the correlation dimension is proposed based on U-statistics, and compared with the weighted least squares estimator proposed by Kawaguchi and Yanagawa (2001). The proposed estimator is easier and faster to compute and has weaker mathematical assumption than the weighted least squares estimator. Moreover, it is shown by simulation that the proposed estimator provides more stable estimates than the weighted least squares estimator when the round-off error of the computer is taken into account.
\end{abstract}

Key Words and Phrases: Chaotic dynamical systems, Correlation dimension, U-statistics, Computer round-off error

\section{Introduction}

We consider trajectory $\left\{X_{t}\right\}_{t=1,2, \ldots, N}$ generated by chaotic dynamical system

$$
X_{t}=F\left(X_{t-1}, X_{t-2}, \ldots, X_{t-d}\right)
$$

for some unknown nonlinear map $F$ and integer $d$. We assume that initial vector $\left(X_{1}, X_{2}, \ldots, X_{d}\right)$ is distributed uniformly in a specified interval. Putting $Y_{t}=\left(X_{t}, X_{t-1}\right.$, $\left.\ldots, X_{t-(d-1)}\right)$ and

$$
C_{N}(r)=\left(\begin{array}{c}
N \\
2
\end{array}\right)^{-1} \sum_{i<j}^{N} I\left(\left\|Y_{i}-Y_{j}\right\| \leq r\right),
$$

where $I$ denotes a indicator function and $\|\cdot\|$ is a norm, Grassberger and Procaccia (abridged by G-P) $(1983 \mathrm{a}, \mathrm{b})$ called $C(r):=\lim _{N \rightarrow \infty} C_{N}(r)$ the correlation integral and introduced the correlation dimension as

$$
p=\lim _{r \rightarrow 0} \frac{\log C(r)}{\log r}
$$

if the limit exists. The correlation dimension was introduced as a measure for representing the fractal dimension of the attractor of $\left\{Y_{t}\right\}$. Estimating the dimension of an attractor of chaotic dynamical systems can provide useful, even vital information for understanding the dynamical systems (see for example, Abraham et al. 1989).

\footnotetext{
- Graduate School of Mathematics, Kyushu University Hakozaki 6-10-1 Higashi-Ku Fukuoka 812-8581

Japan. Email: atsushi@math.kyushu-u.ac.jp
} 
The G-P proposed a procedure for estimating the correlation dimension. It essentially consists of plotting $\log C_{N}(r)$ against $\log r$ and looking for a portion over which the plot is approximately linear, the slope over that portion is the estimator of dimension p. Typically such a graph for a finite length trajectory looks like Figure 1. The graph shows that for large $r$ the graph flattens, at moderate $r$ the graph is quite linear and for small $r$ the graph jumps irregularly. The irregularity is a result of having only a finite amount of data. The linear part of data is often called the scaling region.

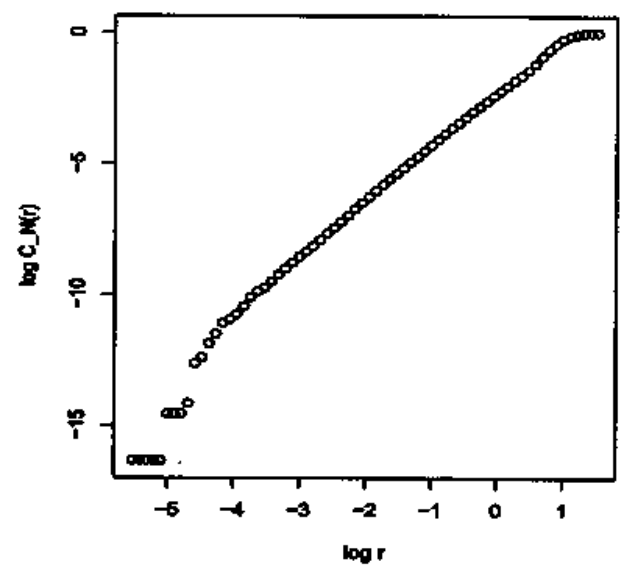

Figure 1: $\log -\log$ plot

The idea of G-P was mathematically realized by Cutler(1990) who proposed a least squares estimator of $p$ over the scaling region taking into account the intercept and by setting

$$
r=r_{0}, r_{1}, \ldots, r_{M}
$$

where $r_{m}=r_{0} \phi^{m}(m=0,1, \ldots, M)$ for some $r_{0}, 0<\phi<1$ and $M=\max \left\{m ; C_{N}\left(r_{m}\right)\right.$ $\neq 0\}$.

It is well known (see, for example, Judd, 1992) that the estimator depends sensitively on the selection of the scaling region. In order to avoid this problem for ordinary least squares, Takens (1984) introduced maximum-likelihood approach. Smith(1992) developed statistical theory by assuming that interpoint distances which are less than $r$ are independent conditioned on distances which are less than $\varepsilon$ for some $\varepsilon$, and proposed an estimator which is essentially equal to Takens' estimator. Confidence intervals for the correlation dimension $p$ was also proposed in Smith(1992). Judd(1992) improved the estimator and confidence interval of Smith(1992) in the same framework as Smith (1992), but with different mathematical manifestation. Kawaguchi and Yanagawa(2001) suggested to use a weighted least square estimator in the formulation of Cutler(1990). The independence of $X_{t}(t=1,2, \ldots, N)$ was assumed there to obtain the weight. The estimator was compared with Cutler's and Smith's estimator. It was shown by simulation that the impact of scaling region on the proposed estimator is smaller than the others. Kawaguchi and Yanagawa(2001) showed numerically that interval by Smith(1992) and 
Judd(1992) failed to take into account the variability due to initial condition when data are generated by known dynamical systems.

The purpose of this paper is to suggest an alternative estimator. The estimator is constructed by using the ordinary method of least squares, but the data are selected by means of U-statistics. The selection enables us to treat the data as homogeneous and frees us from the assumption of independence. As the weighted least squares estimator, the proposed estimator is sensitive to the initial condition caused by round-off error. Thus we introduce a precision interval to compare the estimates. It is shown by simulation that the proposed estimator provides similar estimates as the weighted least squares estimator, and narrower precision intervals than those of the weighted least squares estimator. Moreover, it is easier and faster in computation than the weighted least squares estimator. In Section 2, the estimator is developed. In Section 3, the sensitivity of proposed estimator is shown by simulation and the precision interval is introduced. The estimator is compared with the weighted least squares estimator.

\section{A new estimator based on U-statistics}

We propose to replace $M$ given in (2) with $M^{*}=\max \left\{m ; C_{N 2}\left(r_{m}\right) \neq 0\right\}$, where

$$
C_{N 2}\left(r_{m}\right)=\left(\begin{array}{c}
N \\
3
\end{array}\right)^{-1} \sum_{i \neq j, i \neq k, j \neq k}^{N} I\left(\left\|Y_{i}-Y_{j}\right\| \leq r_{m},\left\|Y_{k}-Y_{j}\right\| \leq r_{m}\right)
$$

Let $i_{1}=\left[M^{*} / 2\right], i_{2}=\left[M^{*} / 2\right]+1, \ldots, i_{L}=M^{*}$. The corresponding model is

$$
\log C_{N}\left(r_{m}\right)=q+p \log r_{m}+e_{m}, \quad\left(m=i_{1}, i_{2}, \ldots, i_{L}\right)
$$

where $e_{m}$ 's are error random variables, satisfying $E\left[e_{m}\right]=0$ and $V\left[e_{m}\right]=\sigma^{2}$. By minimizing

$$
Q=\sum_{m=i_{1}}^{i_{L}}\left(v_{m}-q-p u_{m}\right)^{2}
$$

over all passible choices of $q$ and $p$, where $u_{m}=\log r_{m}, v_{m}=\log C_{N}\left(r_{m}\right)$, and $r_{m}=$ $r_{0} \phi^{m}$, then the estimator of $p$ is explicitly given by

$$
\hat{p}=\frac{\sum_{m=i_{Y}}^{i_{L}}\left(u_{m}-\bar{u}\right)\left(v_{m}-\bar{v}\right)}{\sum_{m=i_{1}}^{i_{L}}\left(u_{m}-\bar{u}\right)^{2}},
$$

where $\vec{u}=L^{-1} \sum_{m=i_{1}}^{i_{L}} u_{m}, \vec{v}=L^{-1} \sum_{m=i_{1}}^{i_{L}} v_{m}$, and $L=M^{*}-\left[M^{*} / 2\right]+1$. It is clear that $\hat{p}$ is unbiased under model (4).

\section{Numerical study}

\subsection{Simulation in floating point arithmetic}

We show by simulation that the estimator proposed is sensitive due to initial condition caused by round-off error when data are generated by known dynamical systems, using the following dynamics. 
(Dy1) $X_{t}=1-1.4 X_{t-1}^{2}+0.3 X_{t-2} \quad$ (Henon map)

(Dy2) $X_{t}=0.01 X_{t-1}+4 X_{t-4} \exp \left(-X_{t-4}^{2}\right)$

(Dy3) $X_{t}=0.5 X_{t-1}+\left(-0.5+2 \exp \left(-X_{t-1}^{2}\right)\right) X_{t-7}$

Note that the embedding dimension of (Dy1), (Dy2) and (Dy3) are $d=2,4$, and 7 , respectively.

To study the impact of round-off errors at order $10^{-1}, 10^{-2}, \ldots$, and $10^{-9}$, we introduce domains $D_{0}, D_{1}, \ldots$, and $D_{9}$ as follows; First decide $D_{0}$ for (Dy1), (Dy2) and (Dy3), respectively by

$D_{0}=$ quadrilateral $\mathrm{ABCD}$, where $\mathrm{A}=(-1.33,1.4), \mathrm{B}=(1.32,0.443), \mathrm{C}=(1.245,-0.466)$, $\mathrm{D}=(-1.06,-1.666)$,

$$
D_{0}=\overbrace{[0,2] \times \cdots \times[0,2]}^{4 \text { times }},
$$

and

$$
D_{0}=\overbrace{[-1,1] \times \cdots \times[-1,1]}^{7 \text { times }} \text {. }
$$

Note that the quadrilateral ABCD is known as Henon's domain of convergence (Henon, 1976). Next, select one point randomly, say $\left(X_{1}^{(0)}, X_{2}^{(0)}, \ldots, X_{d}^{(0)}\right)$ from $D_{0}$, then fix it and construct $D_{1}$ as follows

$$
\begin{array}{r}
D_{1}=\left[X_{1}^{(0)}-0.5 \times 10^{-1}, X_{1}^{(0)}+0.5 \times 10^{-1}\right] \times \cdots \\
\quad \times\left[X_{d}^{(0)}-0.5 \times 10^{-1}, X_{d}^{(0)}+0.5 \times 10^{-1}\right] .
\end{array}
$$

$D_{1}$ shows the interval $\left[X_{1}^{(0)}-0.5 \times 10^{-1}, X_{1}^{(0)}+0.5 \times 10^{-1}\right]$ of 1st decimal place in $d-1$ dimensional space. Similarly, the interval $\left[X_{1}^{(0)}-0.5 \times 10^{-j}, X_{1}^{(0)}+0.5 \times 10^{-j}\right]$ of j-th decimal place in $d-1$ dimensional space is defined by

$$
\begin{array}{r}
D_{j}=\left[X_{1}^{(0)}-0.5 \times 10^{-j}, X_{1}^{(0)}+0.5 \times 10^{-j}\right] \times \cdots \\
\times\left[X_{d}^{(0)}-0.5 \times 10^{-j}, X_{d}^{(0)}+0.5 \times 10^{-j}\right] .
\end{array}
$$

The simulation is conducted as follows; Select an initial value from each of $D_{j}$, $j=0,1, \ldots, 9$, randomly; generate the data of size $N_{0}+N$ from dynamics (Dy1), (Dy2) and (Dy3), respectively; abandon the first $N_{0}$ data and compute the estimate by using the remaining data of size $N$. This process is repeated by $K$ times using the initial values that are selected randomly from $D_{j}(j=0,1, \ldots, 9)$. Constants $N_{0}, N$, and $K$ are set as $N_{0}=1000, N=5000$, and $K=100$, respectively.

Figure 2, 3, and 4 exhibit the result of computation in IEEE single precision arithmetic for (Dy1), (Dy2) and (Dy3), respectively. Since the initial values are selected randomly, it is reasonable to anticipate the fluctuations of the estimates. But at $D_{8}$ and $D_{9}$ the fluctuation of estimates is degenerated, showing that they are beyond the single precision. Table 1, 2, and 3 give the minimum, average and maximum values of 
estimates at $D_{0}, D_{1}, \ldots$, and $D_{7}$. It is remarkable to see that those minimum values at $D_{0}, D_{1}, \ldots$, and $D_{7}$ are essentially equal in the tables; and that maximum values at $D_{0}, D_{1}, \ldots$, and $D_{7}$ are also essentially equal.

To study further, we conducted the similar simulation in IEEE double precision using the initial values which are selected randomly from $D_{12}$. Table 4 summarizes the results. Interestingly, the table again shows that the range of estimates due to the fuctuation at the twelfth decimal order is essentially equal to that the zeroth decimal order. This shows a typical phenomena of the sensitive dependence of chaotic dynamical systems on initial values; that is, we can not free from the dependency even the computation is carried out with high precision. If this is the case, the estimate itself is not trustworthy. We propose to evaluate the estimate with an interval, which we call the precision interval. Fortunately, the Table 1, 2, and 3, and 4 show that the minimum (maximum) at $D_{0}$ is almost equal the minimum (maximum) at $D_{7}$ or $D_{12}$, thus we may construct such interval by taking initial values from $D_{0}$. The interval depends on $K$. We used $K=100$ in the simulation. The precision interval with $K=100$ is called $\mathrm{PI}(100)$ in short.
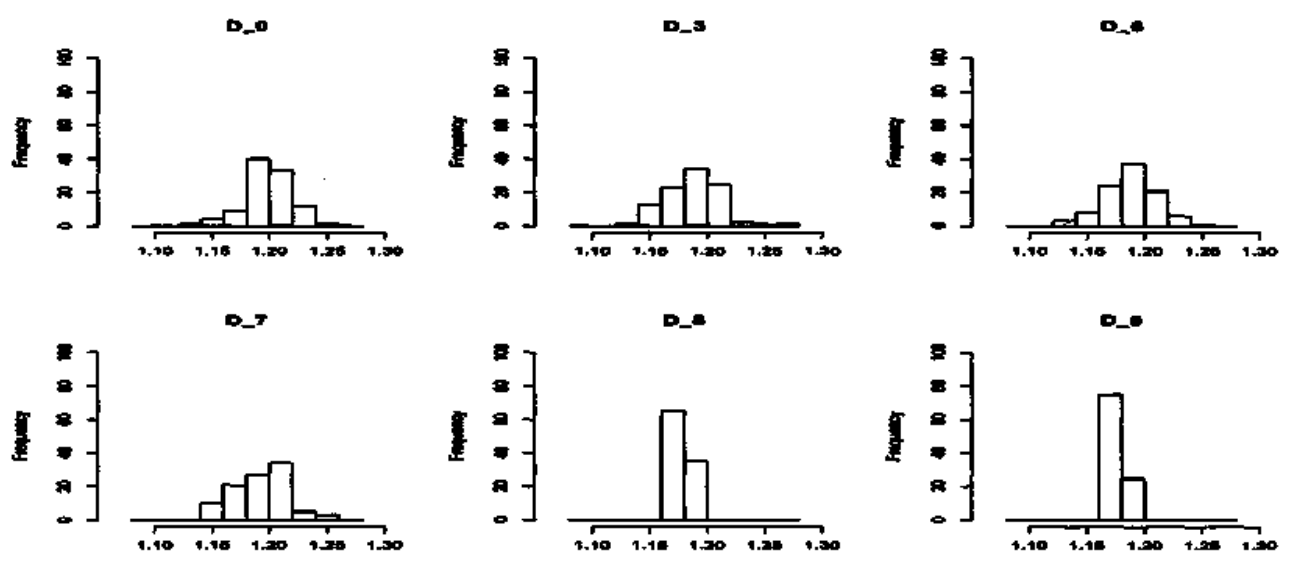

Figure 2: The histgrams for (Dy1)

\begin{tabular}{|r|r|r|r|r|r|r|r|r|}
\hline & $D_{0}$ & $\bar{D}_{1}$ & $\bar{D}_{2}$ & $\overline{D_{3}}$ & $\overline{D_{4}}$ & $\bar{D}_{5}$ & $\bar{D}_{6}$ & $D_{7}$ \\
\hline $\max$ & 1.243 & 1.251 & 1.255 & 1.267 & 1.235 & 1.255 & 1.259 & 1.252 \\
\hline ave & 1.198 & 1.189 & 1.187 & 1.187 & 1.185 & 1.185 & 1.188 & 1.191 \\
\hline $\min$ & 1.137 & 1.130 & 1.098 & 1.125 & 1.114 & 1.116 & 1.126 & 1.144 \\
\hline
\end{tabular}

Table 1: Minimum, average, and maximum values of estimates for (Dy1)

\subsection{Comparison to the weighted least squares estimator}

Simulation is conducted to compare the proposed estimator with the weighted least squares estimator proposed by Kawaguchi and Yanagawa (2001). We recall that the 

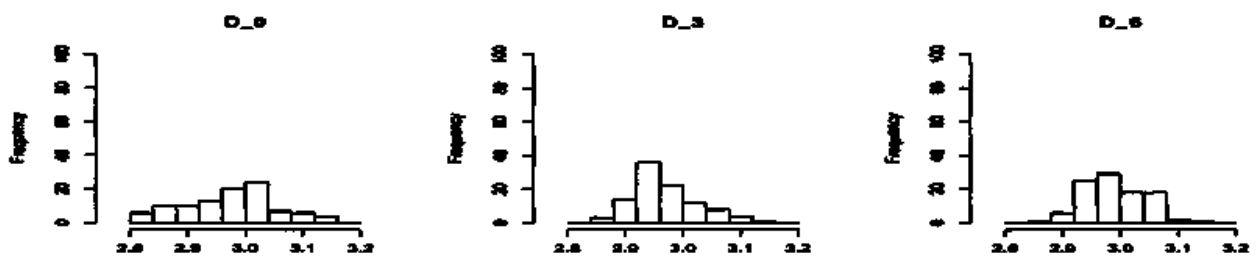

D_?
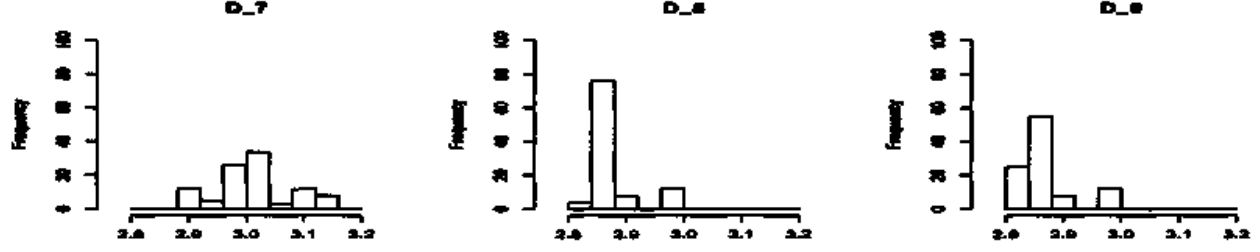

Figure 3: The histgrams for (Dy2)

\begin{tabular}{|r|r|r|r|r|r|r|r|r|}
\hline & $D_{0}$ & $D_{1}$ & $D_{2}$ & $D_{3}$ & $D_{4}$ & $D_{5}$ & $D_{6}$ & $D_{7}$ \\
\hline $\max$ & 3.139 & 3.157 & 3.179 & 3.159 & 3.173 & 3.173 & 3.154 & 3.158 \\
\hline ave & 2.972 & 2.967 & 2.995 & 2.968 & 2.992 & 2.990 & 2.989 & 3.010 \\
\hline $\min$ & 2.807 & 2.810 & 2.860 & 2.855 & 2.821 & 2.864 & 2.871 & 2.881 \\
\hline
\end{tabular}

Table 2: Minimum, average, and maximum values of estimates for (Dy2)
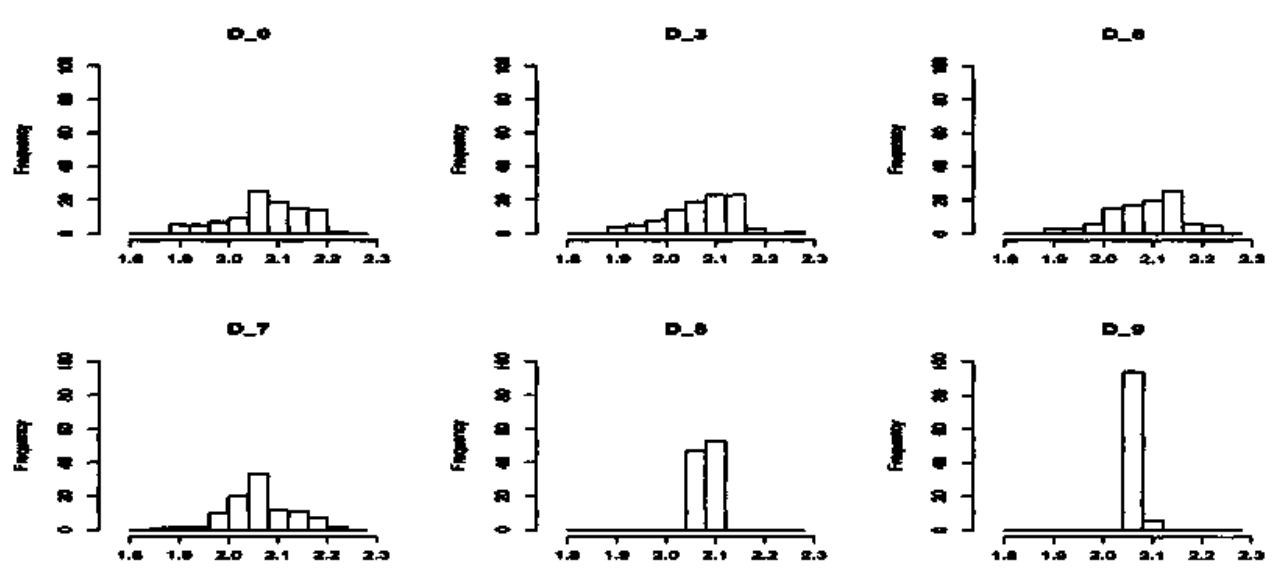

Figure 4: The histgrams for (Dy3)

\begin{tabular}{|r|r|r|r|r|r|r|r|r|}
\hline & $D_{0}$ & $D_{1}$ & $D_{2}$ & $D_{3}$ & $D_{4}$ & $D_{5}$ & $D_{6}$ & $D_{7}$ \\
\hline $\max$ & 2.218 & 2.258 & 2.252 & 2.275 & 2.264 & 2.246 & 2.232 & 2.225 \\
\hline ave & 2.073 & 2.066 & 2.072 & 2.069 & 2.069 & 2.070 & 2.083 & 2.063 \\
\hline $\min$ & 1.886 & 1.884 & 1.880 & 1.897 & 1.869 & 1.887 & 1.886 & 1.854 \\
\hline
\end{tabular}

Table 3: Minimum, average, and maximum values of estimates for (Dy3) 


\begin{tabular}{|c|c|c|c|}
\hline & (Dy1) & (Dy2) & (Dy3) \\
\hline $\max$ & 1.234 & 3.186 & 2.243 \\
\hline ave & 1.190 & 2.989 & 2.094 \\
\hline $\min$ & 1.119 & 2.881 & 1.876 \\
\hline
\end{tabular}

Table 4: Minimum, average, and maximum values of estimates in double precision

weighted least squares estimator was given as follows. For $i_{1}=[M / 2], i_{2}=[M / 2]+1, \ldots$, $i_{L}=M$,

$$
\hat{p}_{w}=\frac{\sum_{m=i_{1}}^{i_{L}} \hat{w}_{m}\left(u_{m}-\bar{u}_{w}\right)\left(v_{m}-\bar{v}_{w}\right)}{\sum_{m=i_{1}}^{i_{L}} \hat{w}_{m}\left(u_{m}-\bar{u}_{w}\right)^{2}},
$$

where $\hat{w}_{m}=\left\{\hat{V}\left[\log C_{N}\left(r_{m}\right)\right]\right\}^{-1}$,

$$
\hat{V}\left[\log C_{N}\left(r_{m}\right)\right]=6\left(\begin{array}{c}
N \\
3
\end{array}\right)\left\{\frac{C_{N 2}\left(r_{m}\right)}{\left(C_{N}\left(r_{m}\right)\right)^{2}}-1\right\}+\left(\begin{array}{c}
N \\
2
\end{array}\right)\left\{\frac{1}{C_{N}\left(r_{m}\right)}-1\right\}
$$

$C_{N 2}\left(r_{m}\right)$ is given in (3), $\bar{u}_{w}=w^{-1} \sum_{m=i_{1}}^{i_{L}} w_{m} u_{m}, \bar{v}_{\mathrm{tw}}=w^{-1} \sum_{m=i_{1}}^{i_{L}} w_{m} v_{m}$, and $w=$ $\sum_{m=i_{1}}^{i_{L}} w_{m}$.

The same size of data i.e. $N=5000$, were generated from (Dy1), (Dy2), and (Dy3), by selecting initial values randomly from $D_{0}$. This process was repeated 100 times. Table 5 summarized the maximum, average, minimum values of 100 estimates obtained respectively by proposed method and weighted lesst squres method.

\begin{tabular}{|r|r|r|r|r|r|r|}
\hline & \multicolumn{2}{|c|}{ (Dy1) } & \multicolumn{2}{c|}{ (Dy2) } & \multicolumn{2}{c|}{ (Dy3) } \\
\hline & proposed & weighted & proposed & weighted & proposed & weighted \\
\hline $\max$ & 1.24 & 1.39 & 3.14 & 3.61 & 2.22 & 2.61 \\
\hline ave & 1.20 & 1.23 & 2.97 & 3.08 & 2.07 & 2.12 \\
\hline min & 1.14 & 0.94 & 2.81 & 2.78 & 1.89 & 1.57 \\
\hline
\end{tabular}

Table 5: Average and PI(100) of proposed estimator (5) and the weighted least squares estimator (6)

The table shows that averages of the proposed estimates and that of the weighted least squares estimates are essentially equal; maximum values of the proposed estimates are smaller than that of the weighted least squares estimates; and that minimum values of the proposed estimates is larger than that of the weighted least squares estimates, that is, the differences between maximum and minimum values of the proposed estimator are smaller than that of the weighted least squares estimator. The table also shows that the difference increases as the increase of the embedding dimension.

\section{References}

Abraham, N. B. Albano, A. M. Passamante, A. and Rapp, P. E. (1989). Measure of@ Complexity and Chaos. New York: Plenum. 
Cutler, C. D. (1990). Some Results on the Behavior and Estimation of the Fractal Dimensions of Distributions on Attractor, Journal of Statistical Physics 62, 651-708.

Grassberger, P. and Procaccia, I. (1983a). Characterization for Strange Attractors,@ Physical Review Letters, 50, No.5, 346-349.

Grassberger, P. and Procaccia, I. (1983b). Measuring the strangeness of strange attractors, Physica D, 9, No.5, 189-208.

Henon, M. (1976). A Two-dimensional Mapping with a Strange Attractor, Communications in Mathematical Physics, 69-77.

Judd, K. (1992). An improved estimator of dimension and some comments on providing confidence intervals, Physica $D, \mathbf{5 6}, 216-228$.

Kawaguchi, A. and Yanagawa, T. (2001). Estimating correlation dimension in chaotic time series. Bulletin of Informatics and Cybernetics, 33, 63-71.

Smith, R. L. (1992). Estimation Dimension in Noisy Chaotic Time Series, Journal of the Royal Statistical Society B, 54, No.2, 329-351.

Takens, F. (1984). On the numerical determination of the dimension of an attractor, Lecture Notes in Mathematics, 1125, 99-106.

Received March 8, 2003 